\title{
On the accuracy of conservation of adiabatic invariants in slow-fast systems
}

\author{
Tan $\mathrm{Su}^{*}$
}

October 24, 2018

\begin{abstract}
Let the adiabatic invariant of action variable in slow-fast Hamiltonian system with two degrees of freedom have two limiting values along the trajectories as time tends to infinity. The difference of two limits is exponentially small in analytic systems. An iso-energetic reduction and canonical transformations are applied to transform the slow-fast systems to form of systems depending on slowly varying parameters in a complexified phase space. On the basis of this method an estimate for the accuracy of conservation of adiabatic invariant is given for such systems.
\end{abstract}

\section{Introduction}

Consider a Hamiltonian system with two degrees of freedom. The Hamiltonian $E$ depends slowly on coordinate $\varepsilon^{-1} x$ and fast on coordinate $q$ :

$$
E=E(p, q, y, x)
$$

where $q, \varepsilon^{-1} x$ are coordinates, and $p, y$ are their conjugate momenta.

The variation of the variables $p, q, y, x$ is described by the differential equations

$$
\dot{p}=-\frac{\partial E}{\partial q}, \quad \dot{q}=\frac{\partial E}{\partial p}, \quad \dot{y}=-\varepsilon \frac{\partial E}{\partial x}, \quad \dot{x}=\varepsilon \frac{\partial E}{\partial y} .
$$

*School of Mathematics, Loughborough University, UK, LE11 3TU 
The variables $p, q$ are called fast, and $y, x$ are called slow variables. This system is called a slow-fast Hamiltonian system. The system with one degree of freedom in which $y=$ const, $x=$ const is called unperturbed or fast system. Assume that when $y, x=$ const, the phase portrait of the unperturbed system contains a domain filled by closed trajectories [1]:

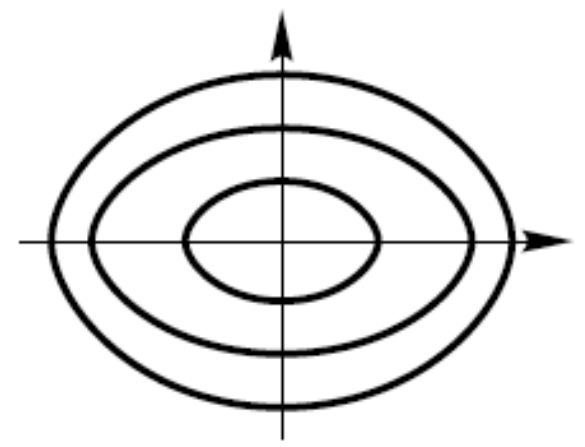

Figure 1

Here the frequency of motion is non-zero. So we can introduce action-angle variables

$$
I=I(p, q, y, x), \quad \varphi=\varphi(p, q, y, x) \quad \bmod 2 \pi
$$

The action $I$ represents the area surrounded by each trajectory. It is equal to this area divided by $2 \pi$. Denote $H_{0}(I, y, x)$ the Hamiltonian $E$ expressed in terms of $I, y, x$. The approximation in which $I=$ const and dynamics of $y, x$ is described by Hamiltonian system with Hamiltonian $H_{0}(I, y, x)$ is called an adiabatic approximation. In this approximation,

$$
\begin{gathered}
E(p, q, y, x) \equiv H_{0}(I, y, x) \\
\dot{y}=-\varepsilon \frac{\partial H_{0}(I, y, x)}{\partial x}, \quad \dot{x}=\varepsilon \frac{\partial H_{0}(I, y, x)}{\partial y} .
\end{gathered}
$$

We will assume that in the phase portrait of this system there is a domain in which along trajectories $|x| \rightarrow \infty, y \rightarrow$ const as time $t \rightarrow \pm \infty$. Thus trajectories have a form shown in one of figures below: 


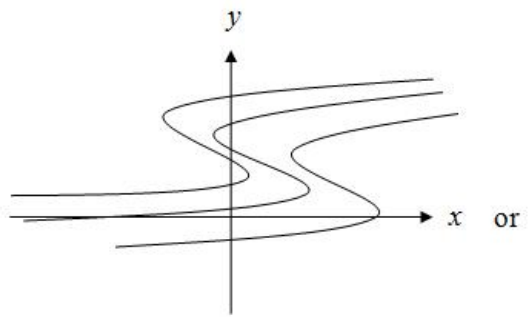

(1)

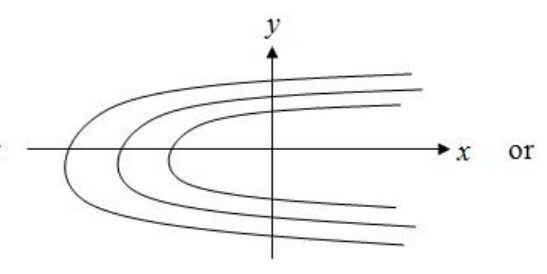

(2)

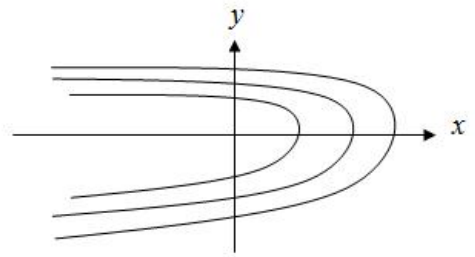

(3)

Figure 2

We consider the case when the adiabatic invariant of the action $I$ along a trajectory of the original system possesses limiting values $I_{ \pm}$as $t \rightarrow \pm \infty$. Their difference $\Delta I=I_{+}-I_{-}$is called an accuracy of persistence of the adiabatic invariant in slow-fast systems.

If this system is analytic, the value of $\Delta I$ is exponentially small [1]:

$$
\Delta I=O\left(\mathrm{e}^{-\frac{\gamma}{\varepsilon}}\right), \quad \gamma=\text { const }>0
$$

The goal of this paper is to find an estimate of constant $\gamma$. After constructing some assumptions, we will then formulate and prove the conclusion.

\section{Reduction to a standard form}

For the original Hamiltonian $E(p, q, y, x)$ with $y, x=$ const, $q$ is the coordinate and $p$ is conjugate momentum. In the unperturbed system, denote $T$ the period of the trajectory and $\omega$ the frequency. Let $S(I, q, y, x)$ denote the generating function of canonical transformation $(p, q) \mapsto(I, \varphi)$. We have the relations [3]:

$$
\varphi=\frac{\partial S(I, q, y, x)}{\partial I}, \quad p=\frac{\partial S(I, q, y, x)}{\partial q}
$$

For a fixed trajectory $E(p, q, y, x)=h=H_{0}(I, y, x)$, where $h$ is a constant, we can express $p$ as

$$
p=P(h, q, y, x)
$$


if $\frac{\partial E}{\partial p} \neq 0$. So the generating function has the following form [3]:

$$
S(I, q, y, x)=\int_{q_{0}}^{q} P\left(h(I, y, x), q_{1}, y, x\right) \mathrm{d} q_{1},
$$

where the constant $q_{0}$ is the initial value of $q_{1}$.

Lemma 2.1. (see, e.g. [4])

$$
\begin{aligned}
& \frac{\partial S}{\partial x}=\int_{0}^{t}\left(\left\langle\frac{\partial E}{\partial x}\right\rangle-\frac{\partial E}{\partial x}\right) \mathrm{d} t_{1}, \\
& \frac{\partial S}{\partial y}=\int_{0}^{t}\left(\left\langle\frac{\partial E}{\partial y}\right\rangle-\frac{\partial E}{\partial y}\right) \mathrm{d} t_{1}
\end{aligned}
$$

where

$$
\left\langle\frac{\partial E}{\partial x}\right\rangle=\frac{1}{T} \oint \frac{\partial E}{\partial x} \mathrm{~d} t_{1}, \quad\left\langle\frac{\partial E}{\partial y}\right\rangle=\frac{1}{T} \oint \frac{\partial E}{\partial y} \mathrm{~d} t_{1} .
$$

Now consider another canonical transformation $(p, q, y, x) \mapsto(\bar{I}, \bar{\varphi}, \bar{y}, \bar{x})$ with generating function $\varepsilon^{-1} \bar{y} x+S(\bar{I}, q, \bar{y}, x)$. The old conjugate variables are $(p, q)$ and $\left(y, \varepsilon^{-1} x\right)$, and new pairs after transformation are $(\bar{I}, \bar{\varphi})$ and $\left(\bar{y}, \varepsilon^{-1} \bar{x}\right)$.

Lemma 2.2. (see, e.g. [1] )

$$
y=\bar{y}+O(\varepsilon), x=\bar{x}+O(\varepsilon), I=\bar{I}+O(\varepsilon), \varphi=\bar{\varphi}+O(\varepsilon)
$$

and new Hamiltonian is

$$
H=H_{0}(\bar{I}, \bar{y}, \bar{x})+\varepsilon H_{1}(\bar{I}, \bar{\varphi}, \bar{y}, \bar{x}, \varepsilon) .
$$

We will consider the case that there exist limiting values of action variables $I$ and $\bar{I}$ as time tends to infinity. The conditions for this and the proof of existence of limits will be added later. Then we will see that the limiting values of $I$ and $\bar{I}$ are equal.

So far we have got a new Hamiltonian

$$
H(\bar{I}, \bar{\varphi}, \bar{y}, \bar{x}, \varepsilon)=H_{0}(\bar{I}, \bar{y}, \bar{x})+\varepsilon H_{1}(\bar{I}, \bar{\varphi}, \bar{y}, \bar{x}, \varepsilon) .
$$

For simplicity, omitting the bar symbols and dependence on $\varepsilon$, we obtain a Hamiltonian system in standard form:

$$
H(I, \varphi, y, x)=H_{0}(I, y, x)+\varepsilon H_{1}(I, \varphi, y, x)
$$


which has motion

$$
\begin{aligned}
& \dot{I}=-\varepsilon \frac{\partial H_{1}}{\partial \varphi}, \quad \dot{\varphi}=\frac{\partial H_{0}}{\partial I}+\varepsilon \frac{\partial H_{1}}{\partial I} \\
& \dot{y}=-\varepsilon\left(\frac{\partial H_{0}}{\partial x}+\varepsilon \frac{\partial H_{1}}{\partial x}\right), \quad \dot{x}=\varepsilon\left(\frac{\partial H_{0}}{\partial y}+\varepsilon \frac{\partial H_{1}}{\partial y}\right) .
\end{aligned}
$$

\section{Statement of the problem}

It is assumed, that in adiabatic approximation, $|x| \rightarrow \infty, y \rightarrow$ const as $t \rightarrow \pm \infty$ (see Figure 2). We will assume also, that when $x, y$ are changing in accordance with adiabatic approximation, and $p, q$ are fixed:

$$
\frac{\partial E(p, q, x, y)}{\partial x} \rightarrow 0, \quad \text { as } t \rightarrow \pm \infty
$$

For definiteness we will consider the case when the trajectories in adiabatic approximation have the form shown in Figure 2(1). Result will be valid for the other two cases of Figure 2.

The function

$$
\omega_{0}(I, y, x)=\frac{\partial H_{0}(I, y, x)}{\partial I}
$$

is the frequency of unperturbed motion.

Let us assume that the following conditions are fulfilled.

\section{Assumption $1^{\circ}$.}

The functions $H_{0}, H_{1}$ can be analytically extended into a complex domain $D=D_{I} \times D_{\varphi} \times D_{x y}$, where $D_{I}$ is a neighbourhood of a given real point $I_{*}$,

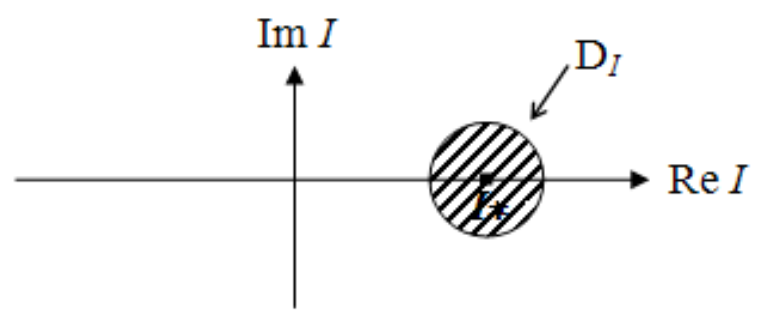

Figure 3 
$D_{\varphi}$ is a strip of a fixed width about the real axis,

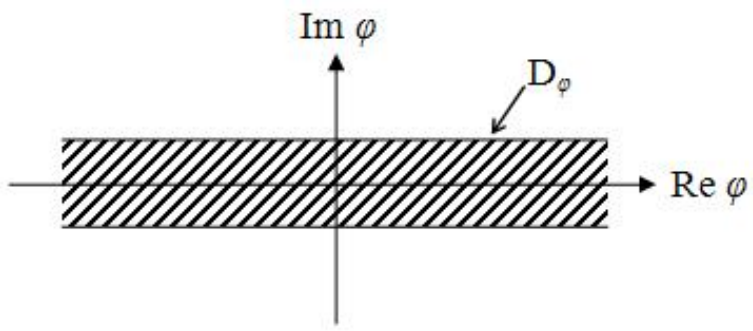

Figure 4

and $D_{x y}$ is some domain in the complex plane $\mathbb{C}^{2}$. The function $\omega_{0}(I, y, x)$ does not vanish in $D$ and $\left|\omega_{0}\right|>$ const. Function $H_{0}(I, y, x)$ satisfies

$$
\left|\frac{\partial H_{0}}{\partial I}\right|<\text { const, } \quad\left|\frac{\partial H_{0}}{\partial y}\right|<\text { const, } \quad\left|\frac{\partial H_{0}}{\partial x}\right|<\text { const, } \quad\left(\frac{\partial H_{0}}{\partial y}\right)^{2}+\left(\frac{\partial H_{0}}{\partial x}\right)^{2}>\text { const. }
$$

Now let us consider approximate Hamiltonian $H_{0}(I, y, x)$ which has motion

$$
\dot{y}=-\varepsilon \frac{\partial H_{0}}{\partial x}, \quad \dot{x}=\varepsilon \frac{\partial H_{0}}{\partial y} .
$$

For a fixed $I_{0}$, we have $H_{0}\left(I_{0}, y, x\right)=h=$ const. Assume that curves $H_{0}=$ const are not closed:

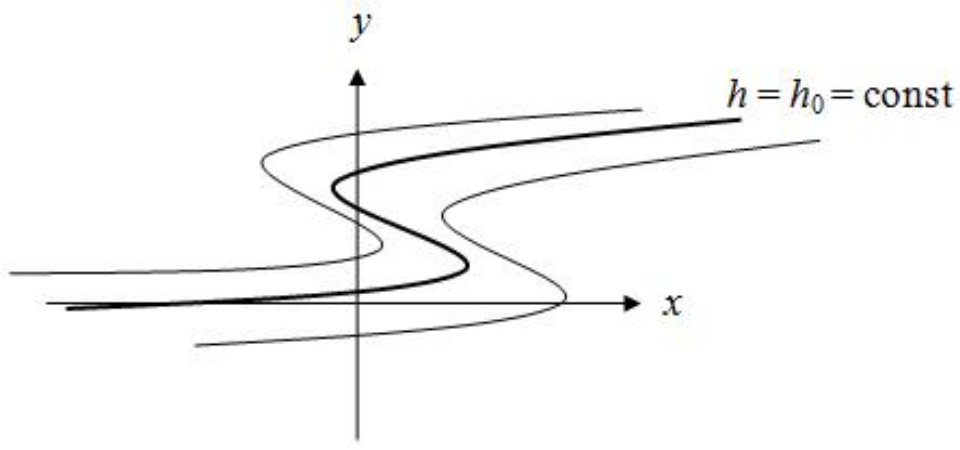

Figure 5

Introduce the slow time

$$
\tau=\varepsilon t .
$$

The differential equations of motion are

$$
\frac{\mathrm{d} y}{\mathrm{~d} \tau}=-\frac{\partial H_{0}}{\partial x}, \quad \frac{\mathrm{d} x}{\mathrm{~d} \tau}=\frac{\partial H_{0}}{\partial y}
$$


Take $h=h_{0}, h_{0}$ is a point in some interval $D_{h}$ with centre $h_{*}$, where $h_{*}$ is a given real point:

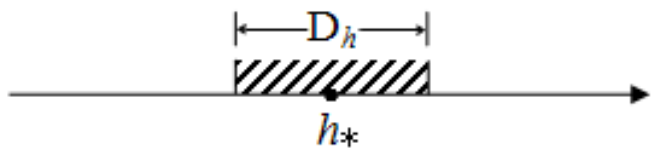

Figure 6

We can find the solution for describing the motion for approximate Hamiltonian $H_{0}$ with $H_{0}\left(I_{0}, y, x\right)=h_{0}$ :

$$
\left\{\begin{array}{l}
y=Y\left(\tau, I_{0}, h_{0}\right) \\
x=X\left(\tau, I_{0}, h_{0}\right)
\end{array} \quad I_{0} \in D_{I}, \quad h_{0} \in D_{h} .\right.
$$

From now on we omit the dependence on $h_{0}$.

\section{Assumption $2^{\circ}$.}

The solutions $Y\left(\tau, I_{0}\right), X\left(\tau, I_{0}\right)$ can be analytically extended into a strip

$$
D_{\tau}=\{\tau:|\operatorname{Im} \tau|<\sigma+\delta|\operatorname{Re} \tau|\} .
$$

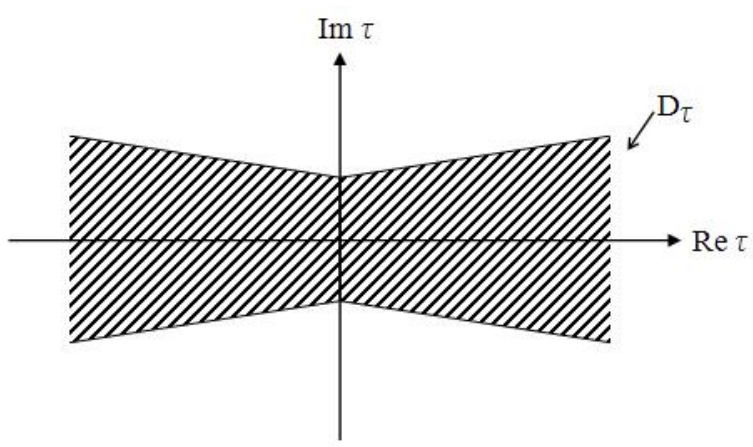

Figure 7

We suppose that for any initial point, the solution tends to infinity as time tends to infinity, i.e.

$$
\lim _{\operatorname{Re} \rightarrow \pm \infty}\left|X\left(\tau, I_{0}\right)\right|=\infty
$$

$H_{0}(I, y, x)$ satisfies

$$
\lim _{\operatorname{Re} \rightarrow \pm \infty} \frac{\partial H_{0}\left(I, Y\left(\tau, I_{0}\right), X\left(\tau, I_{0}\right)\right)}{\partial x}=0
$$


$H_{1}(I, \varphi, y, x)$ satisfies

$$
\left|H_{1}\left(I, \varphi, Y\left(\tau, I_{0}\right), X\left(\tau, I_{0}\right)\right)\right|<\frac{c}{1+\left|\int_{0}^{\tau} \omega_{0}\left(I_{0}, Y\left(\tau_{1}, I_{0}\right), X\left(\tau_{1}, I_{0}\right)\right) \mathrm{d} \tau_{1}\right|^{2+\nu}} .
$$

$\omega_{0}(I, y, x)$ satisfies

$$
\operatorname{Im} \omega_{0}\left(I_{0}, Y\left(\tau, I_{0}\right), X\left(\tau, I_{0}\right)\right) \rightrightarrows 0, \quad \text { as } \operatorname{Re} \tau \rightarrow \pm \infty, \operatorname{Im} I_{0} \rightarrow 0 .
$$

Here $\sigma, \delta, c, \nu$ are positive constants.

Lemma 3.1. For $I \in \widetilde{D}_{I}=D_{I}-\delta_{I}, \varphi \in \widetilde{D}_{\varphi}=D_{\varphi}-\delta_{\varphi}$, and $\tau \in \widetilde{D}_{\tau}=\left\{\tau:|\operatorname{Im} \tau|<\sigma-\delta_{\tau}+\delta|\operatorname{Re} \tau|\right\}$

$$
\begin{aligned}
\left|\frac{\partial H_{1}\left(I, \varphi, Y\left(\tau, I_{0}\right), X\left(\tau, I_{0}\right)\right)}{\partial I}\right| & <\frac{\text { const }}{1+\left|\int_{0}^{\tau} \omega_{0}\left(I_{0}, Y\left(\tau_{1}, I_{0}\right), X\left(\tau_{1}, I_{0}\right)\right) \mathrm{d} \tau_{1}\right|^{2+\nu}}, \\
\left|\frac{\partial H_{1}\left(I, \varphi, Y\left(\tau, I_{0}\right), X\left(\tau, I_{0}\right)\right)}{\partial \varphi}\right| & <\frac{\text { const }}{1+\left|\int_{0}^{\tau} \omega_{0}\left(I_{0}, Y\left(\tau_{1}, I_{0}\right), X\left(\tau_{1}, I_{0}\right)\right) \mathrm{d} \tau_{1}\right|^{2+\nu}}, \\
\left|\frac{\partial H_{1}\left(I, \varphi, Y\left(\tau, I_{0}\right), X\left(\tau, I_{0}\right)\right)}{\partial \tau}\right| & <\frac{\text { const }}{1+\left|\int_{0}^{\tau} \omega_{0}\left(I_{0}, Y\left(\tau_{1}, I_{0}\right), X\left(\tau_{1}, I_{0}\right)\right) \mathrm{d} \tau_{1}\right|^{2+\nu}},
\end{aligned}
$$

where $\delta_{I}, \delta_{\varphi}, \delta_{\tau}$ are positive constants.

Proof. $\operatorname{Im} \omega_{0}\left(I_{0}, Y\left(\tau, I_{0}\right), X\left(\tau, I_{0}\right)\right) \rightrightarrows 0, \quad$ as $\operatorname{Re} \tau \rightarrow \pm \infty, \operatorname{Im} I_{0} \rightarrow 0$ implies that $\forall \rho>0, \exists \Sigma>0, \Gamma>0$, such that if $\left|\operatorname{Im} I_{0}\right|<\Sigma$, $|\operatorname{Re} \tau|>\Gamma$, then

$$
\left|\operatorname{Im} \omega_{0}\right|<\rho .
$$

Since we know that $\left|\omega_{0}\right|>c_{1}=$ const, assume $\left|\operatorname{Im} \omega_{0}\right|<\frac{c_{1}}{10}$ for $\left|\operatorname{Im} I_{0}\right|<\Sigma,|\operatorname{Re} \tau|>\Gamma$, and therefore,

$$
\left|\operatorname{Re} \omega_{0}\right|>\frac{9}{10} c_{1} .
$$

Let $\tau=\lambda+\mathrm{i} \mu$. The integration $\left|\int_{0}^{\tau} \omega_{0}\left(I_{0}, Y\left(\tau_{1}, I_{0}\right), X\left(\tau_{1}, I_{0}\right)\right) \mathrm{d} \tau_{1}\right|$ does not depend on the path. 
For $|\operatorname{Re} \tau|>\max \left\{\Gamma, 2\left(\frac{9}{10} c_{1}-\rho\right)^{-1}\left(\frac{9}{10} c_{1}+\rho+a+b\right) \Gamma\right\}$, where $a, b$ are positive constants satisfying

$$
\int_{0}^{\Gamma}\left|\operatorname{Re} \omega_{0}\right| \mathrm{d} \lambda \leq a \cdot \Gamma, \quad \int_{0}^{\Gamma}\left|\operatorname{Im} \omega_{0}\right| \mathrm{d} \lambda \leq b \cdot \Gamma,
$$

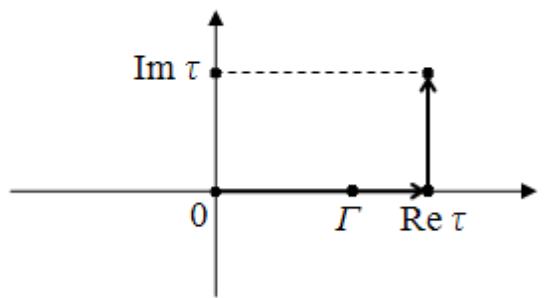

Figure 8

we have

$$
\begin{aligned}
\left|\int_{0}^{\tau} \omega_{0} \mathrm{~d} \tau_{1}\right|= & \left|\int_{0}^{\operatorname{Re} \tau} \omega_{0} \mathrm{~d} \tau_{1}+\int_{\operatorname{Re} \tau}^{\tau} \omega_{0} \mathrm{~d} \tau_{1}\right| \\
= & \left|\int_{0}^{\operatorname{Re} \tau} \omega_{0} \mathrm{~d}(\lambda+\mathrm{i} \mu)+\int_{\operatorname{Re} \tau}^{\tau} \omega_{0} \mathrm{~d}(\lambda+\mathrm{i} \mu)\right| \\
= & \left|\int_{0}^{\operatorname{Re} \tau} \omega_{0} \mathrm{~d} \lambda+\int_{\operatorname{Re} \tau}^{\tau} \omega_{0} \mathrm{~d}(\mathrm{i} \mu)\right| \\
= & \left|\int_{0}^{\operatorname{Re} \tau} \operatorname{Re} \omega_{0} \mathrm{~d} \lambda+\int_{0}^{\operatorname{Re} \tau} \mathrm{i} \operatorname{Im} \omega_{0} \mathrm{~d} \lambda+\int_{\operatorname{Re} \tau}^{\tau} \operatorname{Re} \omega_{0} \mathrm{~d}(\mathrm{i} \mu)+\int_{\operatorname{Re} \tau}^{\tau} \mathrm{i} \operatorname{Im} \omega_{0} \mathrm{~d}(\mathrm{i} \mu)\right| \\
= & \mid \int_{0}^{\Gamma} \operatorname{Re} \omega_{0} \mathrm{~d} \lambda+\int_{\Gamma}^{\operatorname{Re} \tau} \operatorname{Re} \omega_{0} \mathrm{~d} \lambda+\int_{0}^{\tau} \mathrm{i} \operatorname{Im} \omega_{0} \mathrm{~d} \lambda+\int_{\Gamma}^{\mathrm{i} \operatorname{Im} \omega_{0} \mathrm{~d} \lambda} \\
& +\int_{\operatorname{Re} \tau}^{\tau} \operatorname{Re} \omega_{0} \mathrm{~d}(\mathrm{i} \mu)+\int_{\operatorname{Re} \tau}^{\tau} \mathrm{i} \operatorname{Im} \omega_{0} \mathrm{~d}(\mathrm{i} \mu) \mid
\end{aligned}
$$




$$
\begin{aligned}
& \geq|| \int_{\Gamma}^{\operatorname{Re} \tau} \operatorname{Re} \omega_{0} \mathrm{~d} \lambda+\int_{\operatorname{Re} \tau}^{\tau} \operatorname{Re} \omega_{0} \mathrm{~d}(\mathrm{i} \mu)|-| \int_{\Gamma}^{\operatorname{Re} \tau}\left|\operatorname{Im} \omega_{0}\right| \mathrm{d} \lambda+\int_{\operatorname{Re} \tau}^{\tau}\left|\operatorname{Im} \omega_{0}\right| \mathrm{d}(\mathrm{i} \mu) \\
&+\int_{0}^{\Gamma}\left|\operatorname{Re} \omega_{0}\right| \mathrm{d} \lambda+\int_{0}^{\Gamma}\left|\operatorname{Im} \omega_{0}\right| \mathrm{d} \lambda|| \\
&>|| \frac{9}{10} c_{1} \cdot(\operatorname{Re} \tau-\Gamma)+\frac{9}{10} c_{1} \cdot \mathrm{i} \operatorname{Im} \tau|-| \rho \cdot(\operatorname{Re} \tau-\Gamma)+\rho \cdot \mathrm{i} \operatorname{Im} \tau+a \Gamma+b \Gamma|| \\
&=|| \frac{9}{10} c_{1} \cdot \tau-\frac{9}{10} c_{1} \cdot \Gamma|-| \rho \cdot \tau-\rho \cdot \Gamma+a \Gamma+b \Gamma|| \\
& \geq\left|\left(\frac{9}{10} c_{1}|\tau|-\frac{9}{10} c_{1} \Gamma\right)-(\rho|\tau|+\rho \Gamma+a \Gamma+b \Gamma)\right| \\
&=\left|\left(\frac{9}{10} c_{1}-\rho\right)\right| \tau\left|-\left(\frac{9}{10} c_{1}+\rho+a+b\right) \Gamma\right| \\
&>\left(\frac{9}{10} c_{1}-\rho\right)|\tau| \\
& \text { as }\left(\frac{9}{10} c_{1}-\rho\right)|\tau|>2\left(\frac{9}{10} c_{1}+\rho+a+b\right) \Gamma .
\end{aligned}
$$

We can find a positive constant $c_{2}<\frac{9}{10} c_{1}-\rho$, such that

$$
\left|\int_{0}^{\tau} \omega_{0}\left(I_{0}, Y\left(\tau_{1}, I_{0}\right), X\left(\tau_{1}, I_{0}\right)\right) \mathrm{d} \tau_{1}\right|>\left(\frac{9}{10} c_{1}-\rho\right)|\tau|>c_{2}|\tau| .
$$

For $|\operatorname{Re} \tau| \leq \max \left\{\Gamma, 2\left(\frac{9}{10} c_{1}-\rho\right)^{-1}\left(\frac{9}{10} c_{1}+\rho+a+b\right) \Gamma\right\}$, it is evident that we can find another positive constant $c_{3}$ such that

$$
\left|\int_{0}^{\tau} \omega_{0}\left(I_{0}, Y\left(\tau_{1}, I_{0}\right), X\left(\tau_{1}, I_{0}\right)\right) \mathrm{d} \tau_{1}\right|>c_{3}|\tau| .
$$

So

$$
\begin{aligned}
\left|H_{1}\left(I, \varphi, Y\left(\tau, I_{0}\right), X\left(\tau, I_{0}\right)\right)\right| & <\frac{c}{1+\left|\int_{0}^{\tau} \omega_{0}\left(I_{0}, Y\left(\tau_{1}, I_{0}\right), X\left(\tau_{1}, I_{0}\right)\right) \mathrm{d} \tau_{1}\right|^{2+\nu}} \\
& <\frac{c}{1+(\text { const } \cdot|\tau|)^{2+\nu}} \\
& <\frac{\text { const }}{1+|\tau|^{2+\nu}} .
\end{aligned}
$$


From Cauchy estimate [5], we can easily get that, in $\widetilde{D}_{I}$ and $\widetilde{D}_{\varphi}$,

$$
\begin{aligned}
& \left|\frac{\partial H_{1}\left(I, \varphi, Y\left(\tau, I_{0}\right), X\left(\tau, I_{0}\right)\right)}{\partial I}\right|<\frac{\text { const }}{1+|\tau|^{2+\nu}}, \\
& \left|\frac{\partial H_{1}\left(I, \varphi, Y\left(\tau, I_{0}\right), X\left(\tau, I_{0}\right)\right)}{\partial \varphi}\right|<\frac{\text { const }}{1+|\tau|^{2+\nu}} .
\end{aligned}
$$

Now let us prove $\left|\frac{\partial H_{1}\left(I, \varphi, Y\left(\tau, I_{0}\right), X\left(\tau, I_{0}\right)\right)}{\partial \tau}\right|<\frac{\text { const }}{1+|\tau|^{2+\nu}}$. From

$$
D_{\tau}=\{\tau:|\operatorname{Im} \tau|<\sigma+\delta|\operatorname{Re} \tau|\},
$$

for simplicity, assume $\delta_{\tau}=\frac{\sigma}{10}$, and then

$$
\widetilde{D}_{\tau}=\left\{\tau:|\operatorname{Im} \tau|<\sigma-\frac{\sigma}{10}+\delta|\operatorname{Re} \tau|\right\} .
$$

In the following figure, mark the boundary of $D_{\tau}$ with solid lines, and that of $\widetilde{D}_{\tau}$ with dashed lines:

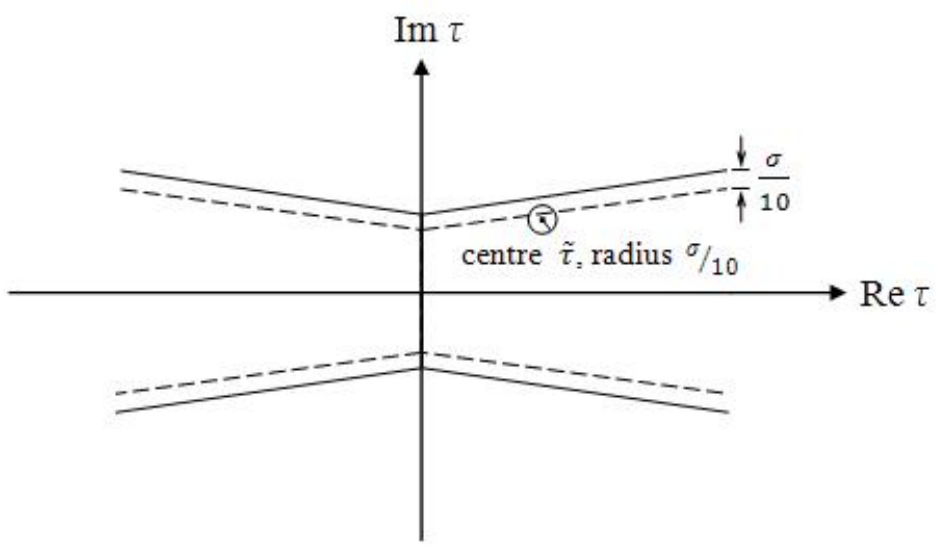

Figure 9

For $\widetilde{\tau} \in \widetilde{D}_{\tau}$, the estimate of $\left|H_{1}\right|$ on the circle of radius $\frac{\sigma}{10}$ around $\widetilde{\tau}$ is

$$
\begin{aligned}
& \left|H_{1}\right|<\frac{\text { const }}{1+\min _{\theta}\left|\widetilde{\tau}+\frac{\sigma}{10} \mathrm{e}^{\mathrm{i} \theta}\right|^{2+\nu}} \text {. If }|\widetilde{\tau}|>\sigma \text {, then } \\
& \qquad\left|H_{1}\right|<\frac{\text { const }}{1+\min _{\theta}\left|\widetilde{\tau}+\frac{\sigma}{10} \mathrm{e}^{\mathrm{i} \theta}\right|^{2+\nu}}<\frac{\text { const }}{1+\left|\frac{9}{10} \widetilde{\tau}\right|^{2+\nu}} .
\end{aligned}
$$


So, because of the Cauchy estimate

$$
\left.\left|\frac{\partial H_{1}}{\partial \tau}\right|\right|_{\tilde{\tau}}<\frac{\text { const }}{1+\left|\frac{9}{10} \widetilde{\tau}\right|^{2+\nu}} / \frac{\sigma}{10}<\frac{\text { const }}{1+|\widetilde{\tau}|^{2+\nu}} .
$$

If $|\widetilde{\tau}| \leq \sigma$, then because of the Cauchy estimate,

$$
\left.\left|\frac{\partial H_{1}}{\partial \tau}\right|\right|_{\widetilde{\tau}}<\text { const } / \frac{\sigma}{10}<\frac{\text { const }}{1+|\widetilde{\tau}|^{2+\nu}} .
$$

Therefore,

$$
\left|\frac{\partial H_{1}\left(I, \varphi, Y\left(\tau, I_{0}\right), X\left(\tau, I_{0}\right)\right)}{\partial \tau}\right|<\frac{\text { const }}{1+|\tau|^{2+\nu}} .
$$

We can find a constant $c_{4}$ such that

$$
\left|\int_{0}^{\tau} \omega_{0}\left(I_{0}, Y\left(\tau_{1}, I_{0}\right), X\left(\tau_{1}, I_{0}\right)\right) \mathrm{d} \tau_{1}\right|<c_{4}|\tau| .
$$

Then

$$
\begin{aligned}
\frac{\text { const }}{1+|\tau|^{2+\nu}} & <\frac{\text { const }}{1+\left(c_{4}^{-1}\left|\int_{0}^{\tau} \omega_{0}\left(I_{0}, Y\left(\tau_{1}, I_{0}\right), X\left(\tau_{1}, I_{0}\right)\right) \mathrm{d} \tau_{1}\right|\right)^{2+\nu}} \\
& <\frac{\text { const }}{1+\left|\int_{0}^{\tau} \omega_{0}\left(I_{0}, Y\left(\tau_{1}, I_{0}\right), X\left(\tau_{1}, I_{0}\right)\right) \mathrm{d} \tau_{1}\right|^{2+\nu}} .
\end{aligned}
$$

Therefore, for $I \in \widetilde{D}_{I}, \varphi \in \widetilde{D}_{\varphi}, \tau \in \widetilde{D}_{\tau}$,

$$
\begin{aligned}
\left|\frac{\partial H_{1}\left(I, \varphi, Y\left(\tau, I_{0}\right), X\left(\tau, I_{0}\right)\right)}{\partial I}\right| & <\frac{\text { const }}{1+\left|\int_{0}^{\tau} \omega_{0}\left(I_{0}, Y\left(\tau_{1}, I_{0}\right), X\left(\tau_{1}, I_{0}\right)\right) \mathrm{d} \tau_{1}\right|^{2+\nu}}, \\
\left|\frac{\partial H_{1}\left(I, \varphi, Y\left(\tau, I_{0}\right), X\left(\tau, I_{0}\right)\right)}{\partial \varphi}\right| & <\frac{\text { const }}{1+\left|\int_{0}^{\tau} \omega_{0}\left(I_{0}, Y\left(\tau_{1}, I_{0}\right), X\left(\tau_{1}, I_{0}\right)\right) \mathrm{d} \tau_{1}\right|^{2+\nu}}, \\
\left|\frac{\partial H_{1}\left(I, \varphi, Y\left(\tau, I_{0}\right), X\left(\tau, I_{0}\right)\right)}{\partial \tau}\right| & <\frac{\text { const }}{1+\left|\int_{0}^{\tau} \omega_{0}\left(I_{0}, Y\left(\tau_{1}, I_{0}\right), X\left(\tau_{1}, I_{0}\right)\right) \mathrm{d} \tau_{1}\right|^{2+\nu}} .
\end{aligned}
$$




\section{Assumption $3^{\circ}$.}

The level lines

$$
\operatorname{Im} \int_{0}^{\tau} \omega_{0}\left(I, Y\left(\tau_{1}, I\right), X\left(\tau_{1}, I\right)\right) \mathrm{d} \tau_{1}=B=\text { const }, \quad 0 \leq|B| \leq \gamma, \quad I \in D_{I}
$$

lie in the domain $D_{\tau}$ and have a positive distance from the boundary of $D_{\tau}$.

Now consider the exact solution $I(t), \varphi(t), y(t), x(t)$ of the Hamiltonian system with Hamiltonian

$$
H(I, \varphi, y, x)=H_{0}(I, y, x)+\varepsilon H_{1}(I, \varphi, y, x)
$$

with real initial conditions $I(0), \varphi(0), y(0), x(0)$ in $D=D_{I} \times D_{\varphi} \times D_{x y}$ at

$t=\tau / \varepsilon=0$, and $H(I(0), \varphi(0), y(0), x(0))=h_{0}$. The adiabatic invariant of the action $I(t)$ satisfies

$$
\frac{\mathrm{d} I}{\mathrm{~d} t}=-\varepsilon \frac{\partial H_{1}(I, \varphi, y, x)}{\partial \varphi}
$$

and thus

$$
I(t)=I(0)-\varepsilon \int_{0}^{t} \frac{\partial H_{1}\left(I\left(t_{1}\right), \varphi\left(t_{1}\right), y\left(t_{1}\right), x\left(t_{1}\right)\right)}{\partial \varphi} \mathrm{d} t_{1} .
$$

Theorem 3.1. There exist limiting values

$$
I_{ \pm}=\lim _{t \rightarrow \pm \infty} I(t)
$$

and their difference $\Delta I=I_{+}-I_{-}$satisfies the estimate

$$
\Delta I=O\left(\mathrm{e}^{-\frac{\gamma}{\varepsilon}}\right), \quad \gamma=\text { const }>0
$$

with the constant $\gamma$ introduced in Assumption $3^{\circ}$.

Remark. The method of continuous averaging [8] gives the same estimate for $\gamma$, as the above-stated theorem.

\section{Example}

Paper [7] gives us several examples of slow-fast Hamiltonian systems, in one of which the Hamiltonian is as follows:

$$
H(I, \varphi, y, x)=\omega I+\frac{y^{2}}{2}+V_{0}(x)+\varepsilon g(\varphi) V_{1}(x) .
$$


Here $y, x$ are slow variables and $I, \varphi$ are fast ones. We explicitly treat the case of $V_{0}(x)=\mathrm{e}^{-x}$. Concerning $V_{1}$, we need it to be real analytic and tend to zero as $x \rightarrow+\infty$. For simplicity we choose $V_{1} \equiv \mathrm{e}^{-x}$. The unperturbed Hamiltonian system is

$$
H_{0}(I, y, x)=\omega I+\frac{y^{2}}{2}+V_{0}(x)=\omega I+\frac{y^{2}}{2}+\mathrm{e}^{-x} .
$$

Let $H_{0}(I, y, x)=\omega I+\frac{y^{2}}{2}+\mathrm{e}^{-x}=h_{0}=$ const be the total energy of the unperturbed system. We can draw the phase portrait of the system to describe the motion:

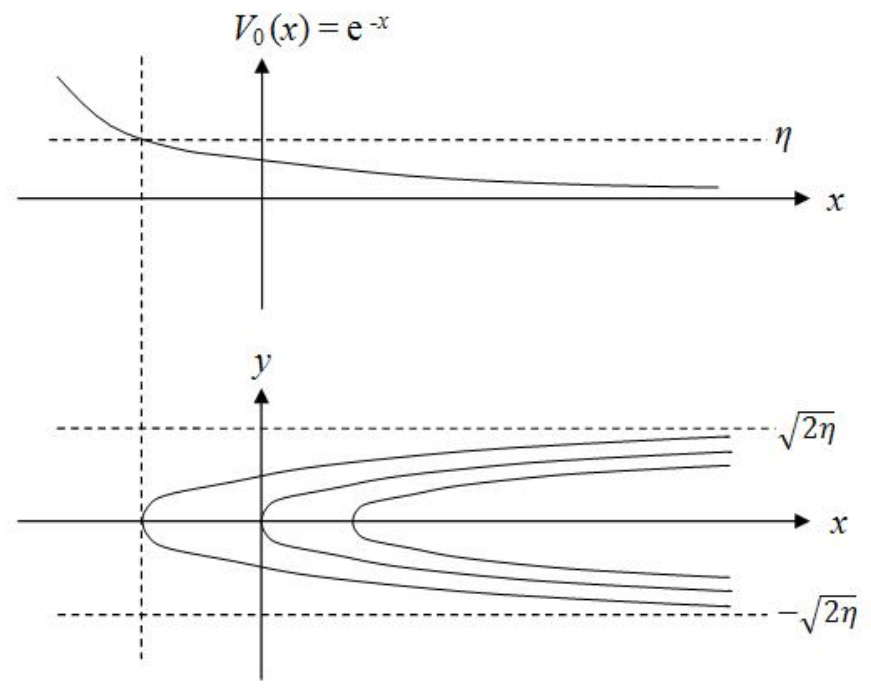

Figure 10

Take $\tilde{\xi}$ as the slow time of motion. From the Hamiltonian $H_{0}(I, y, x)=\omega I+\frac{y^{2}}{2}+\mathrm{e}^{-x}=h_{0}$, we know that

$$
\frac{\mathrm{d} x}{\mathrm{~d} \tilde{\xi}}=\frac{\partial H_{0}}{\partial y}=y
$$

So

$$
\frac{1}{2}\left(\frac{\mathrm{d} x}{\mathrm{~d} \tilde{\xi}}\right)^{2}+\mathrm{e}^{-x}=h_{0}-\omega I=\tilde{\eta} .
$$

Thus we can express $x$ and $y$ via $(\tilde{\xi}, \tilde{\eta})$ :

$$
x=x(\tilde{\xi}, \tilde{\eta}), \quad y=y(\tilde{\xi}, \tilde{\eta})
$$

with initial data $x^{0}=x(0, \tilde{\eta})$ and $y^{0}=\sqrt{2\left(\tilde{\eta}-\mathrm{e}^{-x^{0}}\right)}$.

After solving differential equations, we can obtain the solutions as (see [7])

$$
x(\tilde{\xi}, \tilde{\eta})=\log \left(\frac{1}{\tilde{\eta}}\left(\cosh \sqrt{\tilde{\eta} / 2}\left(\tilde{\xi}-\tilde{\xi}^{0}\right)\right)^{2}\right), \quad y(\tilde{\xi}, \tilde{\eta})=\sqrt{2 \tilde{\eta}} \tanh \sqrt{\tilde{\eta} / 2}\left(\tilde{\xi}-\tilde{\xi}^{0}\right) .
$$


For simplicity we take the initial value of $\tilde{\xi}$ as $\tilde{\xi}^{0}=0$. So $x^{0}=x(0, \tilde{\eta})=\log \frac{1}{\tilde{\eta}}, y^{0}=0$.

Now after the canonical transformation of variables from $(x, y)$ to $(\tilde{\xi}, \tilde{\eta})$, the Hamiltonian $H_{0}$ becomes $K_{0}(\tilde{\xi}, \tilde{\eta})=\omega I+\tilde{\eta}$ and the new Hamiltonian is

$$
K(\tilde{\xi}, \tilde{\eta}, I, \varphi)=\omega I+\tilde{\eta}+\varepsilon f(\tilde{\xi}, \tilde{\eta}) g(\varphi)
$$

with

$$
f(\tilde{\xi}, \tilde{\eta})=V_{1}(x(\tilde{\xi}, \tilde{\eta}))=\mathrm{e}^{-\log \left(\frac{1}{\tilde{\eta}}(\cosh \sqrt{\tilde{\eta} / 2} \tilde{\xi})^{2}\right)}=\frac{\tilde{\eta}}{(\cosh \sqrt{\tilde{\eta} / 2} \tilde{\xi})^{2}} .
$$

The differential equations for describing the motion are

$$
\begin{aligned}
& \dot{\tilde{\xi}}=\varepsilon+\varepsilon^{2} \frac{\partial f(\tilde{\xi}, \tilde{\eta})}{\partial \tilde{\eta}} g(\varphi), \\
& \dot{\tilde{\eta}}=-\varepsilon^{2} \frac{\partial f(\tilde{\xi}, \tilde{\eta})}{\partial \tilde{\xi}} g(\varphi), \\
& \dot{I}=-\varepsilon f(\tilde{\xi}, \tilde{\eta}) \frac{\mathrm{d} g(\varphi)}{\mathrm{d} \varphi}, \\
& \dot{\varphi}=\omega .
\end{aligned}
$$

For $g(\varphi)$, we suppose that $g(\varphi)$ is analytic in the strip $|\operatorname{Im} \varphi|<\rho$ with constant $\rho>0$, and bounded:

$$
|g(\varphi)| \leq 1, \quad \text { for }|\operatorname{Im} \varphi|<\rho
$$

For $f(\tilde{\xi}, \tilde{\eta})$, we can easily prove that

$$
|f(\tilde{\xi}, \tilde{\eta})|<\frac{\text { const }}{1+|\tilde{\xi}|^{2+\nu}}
$$

and

$$
\begin{aligned}
& \left|\frac{\partial f(\tilde{\xi}, \tilde{\eta})}{\partial \tilde{\xi}}\right|<\frac{\mathrm{const}}{1+|\tilde{\xi}|^{2+\nu}}, \\
& \left|\frac{\partial f(\tilde{\xi}, \tilde{\eta})}{\partial \tilde{\eta}}\right|<\frac{\mathrm{const}}{1+|\tilde{\xi}|^{2+\nu}} .
\end{aligned}
$$

Take $D_{I}$ as a neighbourhood of a given real point $I_{*}$, and $D_{\tilde{\varphi}}$ is a strip $|\operatorname{Im} \varphi|<\rho$. Now let us determine the domain of $\tilde{\xi}$ by finding singularities of $f(\tilde{\xi}, \tilde{\eta})$ :

$$
f(\tilde{\xi}, \tilde{\eta})=\frac{\tilde{\eta}}{(\cosh \sqrt{\tilde{\eta} / 2} \tilde{\xi})^{2}}=\frac{4 \tilde{\eta}}{\left(\mathrm{e}^{\sqrt{\tilde{\eta} / 2} \tilde{\xi}}+\mathrm{e}^{-\sqrt{\tilde{\eta} / 2} \tilde{\xi}}\right)^{2}} .
$$


Points of singularities should satisfy

$$
\begin{aligned}
& \mathrm{e}^{\sqrt{\tilde{\eta} / 2} \tilde{\xi}}+\mathrm{e}^{-\sqrt{\tilde{\eta} / 2} \tilde{\xi}}=0 \\
& \mathrm{e}^{2 \sqrt{\tilde{\eta} / 2} \tilde{\xi}}+1=0 \\
& \mathrm{e}^{\sqrt{2 \tilde{\eta}} \tilde{\xi}}=-1 \\
& \mathrm{e}^{\operatorname{Re} \sqrt{2 \tilde{\eta}} \tilde{\xi}}(\cos \operatorname{Im} \sqrt{2 \tilde{\eta}} \tilde{\xi}+\mathrm{i} \sin \operatorname{Im} \sqrt{2 \tilde{\eta}} \tilde{\xi})=-1
\end{aligned}
$$

So $\quad \cos \operatorname{Im} \sqrt{2 \tilde{\eta}} \tilde{\xi}=-\mathrm{e}^{-\operatorname{Re} \sqrt{2 \tilde{\eta}} \tilde{\xi}}<0$, $\sin \operatorname{Im} \sqrt{2 \tilde{\eta}} \tilde{\xi}=0$.

Therefore, $\quad \operatorname{Im} \sqrt{2 \tilde{\eta}} \tilde{\xi}=(2 k+1) \pi, \quad k \in \mathbb{Z}$, and $\operatorname{Re} \sqrt{2 \tilde{\eta}} \tilde{\xi}=0$.

Thus

$$
\sqrt{2 \tilde{\eta}} \tilde{\xi}=(2 k+1) \pi \mathrm{i}, \quad k \in \mathbb{Z} .
$$

The domain of $\tilde{\eta}$ denoted as $D_{\tilde{\eta}}$ is a complex ball around a real value $\tilde{\eta}_{*}$. Any value $\tilde{\eta}^{0} \in D_{\tilde{\eta}}$ can be taken to determine the singularities. Therefore, we obtain the singularities as

$$
\tilde{\xi}=(2 k+1) \frac{\pi}{\sqrt{2 \tilde{\eta}^{0}}} \mathrm{i}, \quad k \in \mathbb{Z} .
$$

From the above conclusion, if we take $\tilde{\xi}$ in the strip

$$
D_{\tilde{\xi}}=\left\{\tilde{\xi}:|\operatorname{Im} \tilde{\xi}|<\frac{\pi}{\sqrt{2 \tilde{\eta}^{0}}}+\delta|\operatorname{Re} \tilde{\xi}|\right\},
$$

where $\delta$ is a positive constant, $f(\tilde{\xi}, \tilde{\eta})$ is analytic for any $\tilde{\eta} \in D_{\tilde{\eta}}$. The following figure shows the boundary of domain $D_{\tilde{\xi}}$ : 


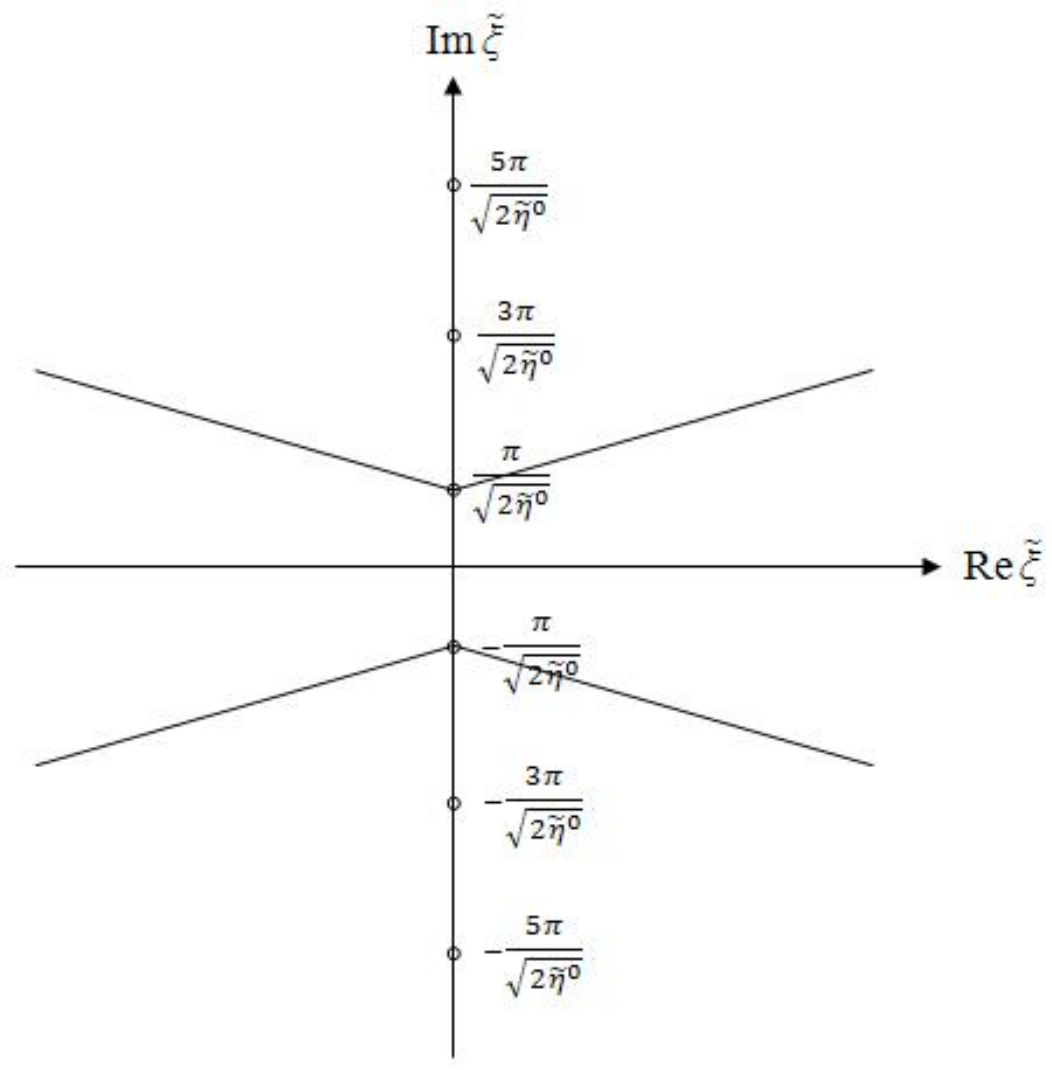

Figure 11

We can find the level lines

$$
\operatorname{Im} \int_{0}^{\tilde{\xi}} \omega \mathrm{d} \tilde{\xi}_{1}=\omega \operatorname{Im} \tilde{\xi}=B=\text { const, } \quad 0 \leq|B| \leq \gamma
$$

lying in the domain $D_{\tilde{\xi}}$, which have a positive distance from the boundary of this domain. So $\gamma$ has been found as any constant such that

$$
\gamma<\frac{\pi \omega}{\sqrt{2 \tilde{\eta}^{0}}}, \quad \text { for such definition of } \tilde{\eta}_{0}
$$

According to Theorem 1, the accuracy of conservation of adiabatic invariant $\Delta I$ has the estimate:

$$
\Delta I=O\left(\mathrm{e}^{-\frac{\gamma}{\varepsilon}}\right)
$$

This estimate coincides with that in [7], where it was obtained by a different method. 


\section{Proof of the Theorem}

The original system is described by Hamiltonian

$$
H(I, \varphi, y, x)=H_{0}(I, y, x)+\varepsilon H_{1}(I, \varphi, y, x)
$$

with motion in time $t$ :

$$
\begin{aligned}
& \dot{I}=-\varepsilon \frac{\partial H_{1}}{\partial \varphi}, \quad \dot{\varphi}=\frac{\partial H_{0}}{\partial I}+\varepsilon \frac{\partial H_{1}}{\partial I} \\
& \dot{y}=-\varepsilon\left(\frac{\partial H_{0}}{\partial x}+\varepsilon \frac{\partial H_{1}}{\partial x}\right), \quad \dot{x}=\varepsilon\left(\frac{\partial H_{0}}{\partial y}+\varepsilon \frac{\partial H_{1}}{\partial y}\right) .
\end{aligned}
$$

As the frequency $\omega_{0}(I, y, x)=\frac{\partial H_{0}(I, y, x)}{\partial I}$ does not vanish in our domain $D$, we can express $I$ from $H$ via $\varphi, y, x$ and a constant of energy. This is the procedure of isoenergetic reduction on the energy level $H(I, \varphi, y, x)=h_{0}, h_{0}=$ const:

$$
-I\left(y, x, \varphi, h_{0}\right)=F_{0}\left(y, x, h_{0}\right)+\varepsilon F_{1}\left(y, x, \varphi, h_{0}\right) .
$$

Here $(-I)$ is the new Hamiltonian, $\varphi$ is the new time, and $(y, x)$ is in a small neighbourhood of $\widetilde{D}_{x y}$, which can be written as $\widetilde{D}_{x y}+\delta_{x y}$, where

$$
\widetilde{D}_{x y}=\left\{(y, x):\left\{\begin{array}{l}
y=Y\left(\tau, I_{0}, h_{0}\right) \\
x=X\left(\tau, I_{0}, h_{0}\right)
\end{array}, \tau \in D_{\tau}, I_{0} \in D_{I}\right\},\right.
$$

and $\delta_{x y}$ is some positive constant.

We do not indicate dependence of $Y, X$ on $\varepsilon$. The differential equations of motion are

$$
\frac{\mathrm{d} y}{\mathrm{~d} \varphi}=-\varepsilon\left(\frac{\partial F_{0}}{\partial x}+\varepsilon \frac{\partial F_{1}}{\partial x}\right), \quad \frac{\mathrm{d} x}{\mathrm{~d} \varphi}=\varepsilon\left(\frac{\partial F_{0}}{\partial y}+\varepsilon \frac{\partial F_{1}}{\partial y}\right) .
$$

Since we know functions $H_{0}$ and $H_{1}$ are analytic in $D$, from the implicit function theorem, functions $F_{0}$ and $F_{1}$ are also analytic in $D_{\varphi} \times\left(\widetilde{D}_{x y}+\delta_{x y}\right)$.

Now consider the approximate system with Hamiltonian

$$
F_{0}\left(y, x, h_{0}\right)=-I_{0}
$$

Introduce slow time $\xi=\varepsilon \varphi$, and the motion is

$$
\frac{\mathrm{d} y}{\mathrm{~d} \xi}=-\frac{\partial F_{0}}{\partial x}, \quad \frac{\mathrm{d} x}{\mathrm{~d} \xi}=\frac{\partial F_{0}}{\partial y} .
$$

We can find the solution for the above approximate system:

$$
\left\{\begin{array}{l}
y=\widehat{Y}\left(\xi, I_{0}, h_{0}\right) \\
x=\widehat{X}\left(\xi, I_{0}, h_{0}\right)
\end{array} \quad I_{0} \in D_{I}, \quad h_{0} \in D_{h} .\right.
$$


In what follows, we do not indicate the dependence on constant $h_{0}$. The relation between solutions $Y\left(\tau, I_{0}\right), X\left(\tau, I_{0}\right)$ and $\widehat{Y}\left(\xi, I_{0}\right), \widehat{X}\left(\xi, I_{0}\right)$ follows from the formula

$$
\xi=\int_{0}^{\tau} \omega_{0}\left(I_{0}, Y\left(\tau_{1}, I_{0}\right), X\left(\tau_{1}, I_{0}\right)\right) \mathrm{d} \tau_{1} .
$$

Solution $\widehat{Y}\left(\xi, I_{0}\right), \widehat{X}\left(\xi, I_{0}\right)$ can be analytically extended into some domain $D_{\xi}$ with respect to $\xi$. The domain $D_{\xi}$ will be introduced in later lemma. As we know,

$$
\operatorname{Im} \omega_{0}\left(I_{0}, Y\left(\tau, I_{0}\right), X\left(\tau, I_{0}\right)\right) \rightrightarrows 0, \quad \text { as } \operatorname{Re} \tau \rightarrow \pm \infty, \operatorname{Im} I_{0} \rightarrow 0 .
$$

It implies that $\omega_{0}$ uniformly tends to a real function depending on $\operatorname{Re} I_{0}$ as time tends to infinity and $I_{0}$ tends to real. Let us denote the limits as $\omega_{ \pm}\left(\operatorname{Re} I_{0}\right)$ :

$$
\omega_{0}\left(I_{0}, Y\left(\tau_{1}, I_{0}\right), X\left(\tau_{1}, I_{0}\right)\right) \rightrightarrows \omega_{ \pm}\left(\operatorname{Re} I_{0}\right), \quad \text { as } \operatorname{Re} \tau \rightarrow \pm \infty, \operatorname{Im} I_{0} \rightarrow 0 .
$$

Lemma 5.1. For $I_{0} \in D_{I}$ close to the imaginary axis, the image of the domain $D_{\tau}$ under the map $\tau \mapsto \xi$ given by the formula

$$
\xi=\int_{0}^{\tau} \omega_{0}\left(I_{0}, Y\left(\tau_{1}, I_{0}\right), X\left(\tau_{1}, I_{0}\right)\right) \mathrm{d} \tau_{1}
$$

is a strip around the real axis that contains a domain

$$
D_{\xi}=\left\{\xi:\left\{\begin{array}{ll}
|\operatorname{Im} \xi|<\sigma_{1}+\delta_{1}|\operatorname{Re} \xi|, & |\operatorname{Re} \xi|>c_{5} \Gamma \\
|\operatorname{Im} \xi|<\sigma_{2}, & |\operatorname{Re} \xi| \leq c_{5} \Gamma
\end{array}\right\}\right.
$$

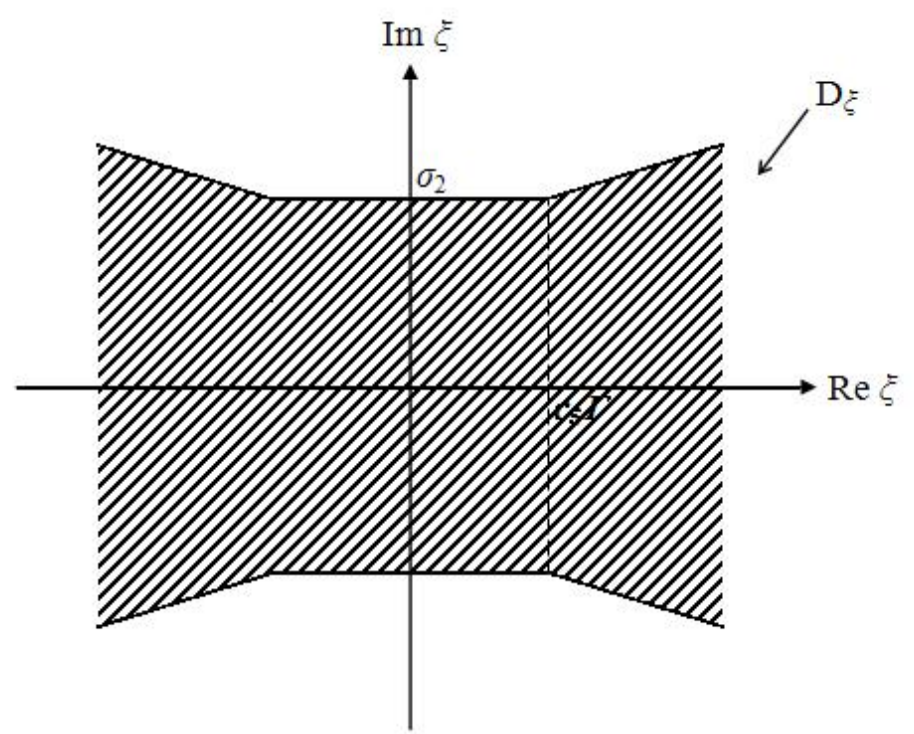

Figure 12 
where $\sigma_{1}, \sigma_{2}, \delta_{1}, c_{5}$ are positive constants and $\sigma_{2}=\sigma_{1}+\delta_{1} \cdot c_{5} \Gamma$ for continuity. Straight lines $\operatorname{Im} \xi= \pm \gamma$ lie in $D_{\xi}$ and have a positive distance from the boundary of $D_{\xi}$ (i.e. $\gamma<\sigma_{2}$ ).

Lemma 5.2. The function $F_{1}(y, x, \varphi)$ satisfies the estimate:

$$
\left|F_{1}\left(\widehat{Y}\left(\xi, I_{0}\right), \widehat{X}\left(\xi, I_{0}\right), \varphi\right)\right|<\frac{\text { const }}{1+|\xi|^{2+\nu}} .
$$

Proof. First of all let us list two forms of Hamiltonian:

$$
\begin{aligned}
& H_{0}(I, y, x)+\varepsilon H_{1}(I, \varphi, y, x)=h_{0}, \\
& -I=F_{0}(y, x)+\varepsilon F_{1}(y, x, \varphi) .
\end{aligned}
$$

Therefore, we have

$$
H_{0}\left(-\left(F_{0}+\varepsilon F_{1}\right), y, x\right)+\varepsilon H_{1}\left(-\left(F_{0}+\varepsilon F_{1}\right), \varphi, y, x\right)=h_{0} .
$$

Then let $\varepsilon=0: \quad H_{0}\left(-F_{0}, y, x\right)=h_{0}$.

So

$$
\begin{aligned}
& H_{0}\left(-\left(F_{0}+\varepsilon F_{1}\right), y, x\right)+\varepsilon H_{1}\left(-\left(F_{0}+\varepsilon F_{1}\right), \varphi, y, x\right)=H_{0}\left(-F_{0}, y, x\right), \\
& H_{0}\left(-\left(F_{0}+\varepsilon F_{1}\right), y, x\right)-H_{0}\left(-F_{0}, y, x\right)=-\varepsilon H_{1}\left(-\left(F_{0}+\varepsilon F_{1}\right), \varphi, y, x\right) .
\end{aligned}
$$

Applying the intermediate value theorem here, we get that there exist some value $\widetilde{I}$, such that

$$
\begin{aligned}
\frac{\partial H_{0}(\widetilde{I}, y, x)}{\partial I} & \cdot\left(-\varepsilon F_{1}\right)=-\varepsilon H_{1}\left(-\left(F_{0}+\varepsilon F_{1}\right), \varphi, y, x\right) \\
\text { so } \quad & F_{1}(y, x, \varphi)=\frac{1}{\frac{\partial H_{0}(\widetilde{I}, y, x)}{\partial I}} \cdot H_{1}(I, \varphi, y, x)
\end{aligned}
$$

We know from assumptions that

$$
\left|\omega_{0}\right|=\left|\frac{\partial H_{0}}{\partial I}\right|>\text { const }
$$

and $\left|H_{1}\left(I, \varphi, Y\left(\tau, I_{0}\right), X\left(\tau, I_{0}\right)\right)\right|<$

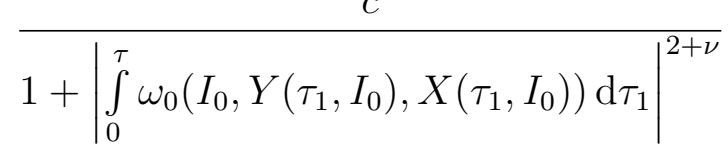

Therefore,

$$
\begin{aligned}
\left|F_{1}\left(\widehat{Y}\left(\xi, I_{0}\right), \widehat{X}\left(\xi, I_{0}\right), \varphi\right)\right| & <\text { const } \cdot\left|H_{1}\left(I, \varphi, Y\left(\tau, I_{0}\right), X\left(\tau, I_{0}\right)\right)\right| \\
& <\frac{\text { const }}{1+|\xi|^{2+\nu}} .
\end{aligned}
$$


Corollary 5.1. For $\varphi \in \widetilde{D}_{\varphi}=D_{\varphi}-\delta_{\varphi}, \xi \in \widetilde{D}_{\xi}=D_{\xi}-\delta_{\xi}$,

$$
\begin{aligned}
& \left|\frac{\partial F_{1}\left(\widehat{Y}\left(\xi, I_{0}\right), \widehat{X}\left(\xi, I_{0}\right), \varphi, h_{0}\right)}{\partial \varphi}\right|<\frac{\text { const }}{1+|\xi|^{2+\nu}}, \\
& \left|\frac{\partial F_{1}\left(\widehat{Y}\left(\xi, I_{0}\right), \widehat{X}\left(\xi, I_{0}\right), \varphi, h_{0}\right)}{\partial \xi}\right|<\frac{\text { const }}{1+|\xi|^{2+\nu}},
\end{aligned}
$$

where $\delta_{\xi}$ is a positive constant.

Now let us draw the phase portrait of $F_{0}(y, x)=-I$ :

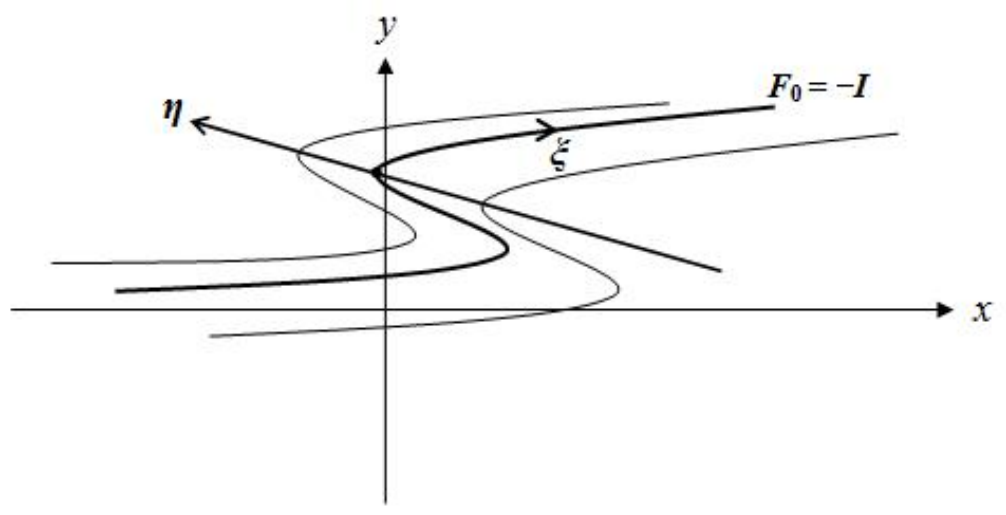

Figure 13

and introduce new coordinates $(\xi, \eta)$. Here $\eta$ is exactly the value of $F_{0}$ on level lines $F_{0}=\eta$, and $\xi$ is the slow time of motion. The initial section is $\widehat{X}(0, I), \widehat{Y}(0, I)$ parametrized by $I$. Then construct a transformation $(\xi, \eta) \mapsto(x, y)$.

Lemma 5.3. In the domain $D_{\xi} \times D_{\eta}$, where $D_{\xi}$ is what mentioned above and $D_{\eta}$ is a neighbourhood of the real point $-I_{*}$, the mapping $(\xi, \eta) \mapsto(x, y)$ is analytic and canonical.

Proof. With map $(\xi, \eta) \mapsto(x, y)$, consider $x$ and $y$ as functions of $\xi, \eta$ :

$$
x=x(\xi, \eta), \quad y=y(\xi, \eta) .
$$

From the motion above, we have obtained the relations:

$$
\frac{\partial x}{\partial \xi}=\frac{\partial F_{0}}{\partial y}, \quad \frac{\partial y}{\partial \xi}=-\frac{\partial F_{0}}{\partial x} .
$$

Let us differentiate both sides of the Hamiltonian $F_{0}\left(y, x, h_{0}\right)=\eta$ with respect to $\eta$ :

$$
\frac{\partial F_{0}}{\partial y} \frac{\partial y}{\partial \eta}+\frac{\partial F_{0}}{\partial x} \frac{\partial x}{\partial \eta}=1
$$


Replacing with corresponding derivatives, we get

$$
\frac{\partial x}{\partial \xi} \frac{\partial y}{\partial \eta}-\frac{\partial y}{\partial \xi} \frac{\partial x}{\partial \eta}=1
$$

That is indeed the Jacobi determinant:

$$
\frac{D(x, y)}{D(\xi, \eta)}=\operatorname{det}\left|\begin{array}{ll}
\frac{\partial x}{\partial \xi} & \frac{\partial x}{\partial \eta} \\
\frac{\partial y}{\partial \xi} & \frac{\partial y}{\partial \eta}
\end{array}\right|=1 .
$$

Therefore, $(x, y) \mapsto(\xi, \eta)$ is canonical transformation.

After this transformation, the new Hamiltonian has the form

$$
-I(\eta, \xi, \varphi)=\eta+\varepsilon G_{1}(\eta, \xi, \varphi) .
$$

Function $G_{1}$ is analytic and has the following estimate:

$$
\left|G_{1}(\eta, \xi, \varphi)\right|<\frac{\text { const }}{1+|\xi|^{2+\nu}} .
$$

Also from Cauchy estimate [5], for $\eta \in \widetilde{D}_{\eta}=D_{\eta}-\delta_{\eta}, \xi \in \widetilde{D}_{\xi}=D_{\xi}-\delta_{\xi}, \varphi \in \widetilde{D}_{\varphi}$, where $\delta_{\eta}$ and $\delta_{\xi}$ are positive constants, we obtain

$$
\begin{aligned}
& \left|\frac{\partial G_{1}(\eta, \xi, \varphi)}{\partial \varphi}\right|<\frac{\text { const }}{1+|\xi|^{2+\nu}}, \\
& \left|\frac{\partial G_{1}(\eta, \xi, \varphi)}{\partial \eta}\right|<\frac{\text { const }}{1+|\xi|^{2+\nu}}, \\
& \left|\frac{\partial G_{1}(\eta, \xi, \varphi)}{\partial \xi}\right|<\frac{\text { const }}{1+|\xi|^{2+\nu}} .
\end{aligned}
$$

Because of the above estimate, we can take another similar isoenergetic reduction through expressing $\eta$, via $I, \varphi, \xi$ and constant $h_{0}$ :

$$
-\eta(I, \varphi, \xi)=I+\varepsilon K_{1}(I, \varphi, \xi) .
$$

Here $(-\eta)$ is the new Hamiltonian, and $\xi$ is the new slow time. $I$ and $\varphi$ are conjugate variables. 
From implicit function theorem, we can obtain the similar conclusions that function $K_{1}$ is analytic in $\widehat{D}_{I} \times D_{\varphi} \times D_{\xi}$ and estimates of $K_{1}$ and its derivatives have the following forms:

$$
\begin{aligned}
& \left|K_{1}(I, \varphi, \xi)\right|<\frac{\text { const }}{1+|\xi|^{2+\nu}}, \\
& \left|\frac{\partial K_{1}(I, \varphi, \xi)}{\partial I}\right|<\frac{\text { const }}{1+|\xi|^{2+\nu}}, \\
& \left|\frac{\partial K_{1}(I, \varphi, \xi)}{\partial \varphi}\right|<\frac{\text { const }}{1+|\xi|^{2+\nu}}, \\
& \left|\frac{\partial K_{1}(I, \varphi, \xi)}{\partial \xi}\right|<\frac{\text { const }}{1+|\xi|^{2+\nu}},
\end{aligned}
$$

in $\left(\widehat{D}_{I}-\delta_{I}\right) \times \widetilde{D}_{\varphi} \times \widetilde{D}_{\xi}$. Here $\widehat{D}_{I}$ is the same as the domain $-D_{\eta}$.

In this latest Hamiltonian, $K_{0} \equiv I$ and the frequency is $\frac{\partial K_{0}}{\partial I}=1$. The level lines

$$
\operatorname{Im} \int_{0}^{\xi} \mathrm{d} \xi_{1}=\operatorname{Im} \xi=B=\text { const }, \quad 0 \leq|B| \leq \gamma
$$

also lie in the domain $D_{\xi}$ and have a positive distance from the boundary of $D_{\xi}$. The constant $\gamma$ is the same as introduced in Assumption $3^{\circ}$.

Denoting the new time as $\vartheta=\varepsilon^{-1} \xi$, we can write differential equations of motion:

$$
\frac{\mathrm{d} I}{\mathrm{~d} \vartheta}=-\varepsilon \frac{\partial K_{1}}{\partial \varphi}, \quad \frac{\mathrm{d} \varphi}{\mathrm{d} \vartheta}=1+\frac{\partial K_{1}}{\partial I} .
$$

Now consider the exact solution $I(\vartheta), \varphi(\vartheta)$ with real initial conditions $I(0), \varphi(0)$ at $\vartheta_{0}=$ $\varepsilon^{-1} \xi_{0}=0$. We obtain the relation which the solution satisfies as follows:

$$
I(\vartheta)=I(0)-\varepsilon \int_{0}^{\vartheta} \frac{\partial K_{1}\left(I\left(\vartheta_{1}\right), \varphi\left(\vartheta_{1}\right), \varepsilon \vartheta_{1}\right)}{\partial \varphi} \mathrm{d} \vartheta_{1} .
$$

From the Cauchy criterion [6], we can easily prove the existence of limiting values of $I(\vartheta)$ as $\vartheta \rightarrow \pm \infty$. Moreover, we also know differential equations of motion:

$$
\begin{aligned}
\frac{\mathrm{d} \varphi}{\mathrm{d} \vartheta} & =1+\varepsilon \frac{\partial K_{1}}{\partial \varphi} \\
\text { and } \quad \frac{\mathrm{d} \varphi}{\mathrm{d} t} & =\frac{\partial H_{0}}{\partial I}+\varepsilon \frac{\partial H_{1}}{\partial I} .
\end{aligned}
$$

It is evident that $\varphi \rightarrow \pm \infty$ as $\vartheta \rightarrow \pm \infty$. Meanwhile, $t \rightarrow \pm \infty$. Therefore, the limits when $t \rightarrow \pm \infty$ are equal to those when $\vartheta \rightarrow \pm \infty$. Let us define values

$$
I_{ \pm}=\lim _{t \rightarrow \pm \infty} I(t), \quad \Delta I=I_{+}-I_{-} .
$$


Now the system under consideration is described by the Hamiltonian

$$
-\eta(I, \varphi, \xi)=I+\varepsilon K_{1}(I, \varphi, \xi),
$$

where $I$ and $\varphi$ are conjugate canonical variables. The action-angle variables $I, \varphi$ of the unperturbed problem are defined in region $\widehat{D}_{I} \times D_{\varphi}$. $\xi$ is the slow time variable defined in region $D_{\xi}$ and time $\vartheta=\varepsilon^{-1} \xi$. The function $K_{1}$ is an analytic, $2 \pi$-periodic in $\varphi$ function. The frequency of unperturbed motion is 1 . We have proved the following properties:

$1^{\circ}$. The functions $K_{0}=I, K_{1}$ can be analytically extended into a complex domain $\widehat{D}_{I} \times$ $D_{\varphi} \times D_{\xi}$, where $\widehat{D}_{I}$ is some neighbourhood of a given real point $I_{*}, D_{\varphi}$ is a strip of some fixed width about the real axis, and $D_{\xi}$ is a strip

$$
D_{\xi}=\left\{\xi:\left\{\begin{array}{ll}
|\operatorname{Im} \xi|<\sigma_{1}+\delta_{1}|\operatorname{Re} \xi|, & |\operatorname{Re} \xi|>c_{5} \Gamma \\
|\operatorname{Im} \xi|<\sigma_{2}, & |\operatorname{Re} \xi| \leq c_{5} \Gamma
\end{array}\right\}\right.
$$

where $\sigma_{1}+\delta_{1} \cdot c_{5} \Gamma=\sigma_{2}$. The function $K_{1}$ satisfies the estimate

$$
\left|K_{1}(I, \varphi, \xi)\right|<\frac{C}{1+|\xi|^{2+\nu}} .
$$

Here $\sigma, \delta, C, \nu$ are positive constant.

$2^{\circ}$. The level lines

$$
\operatorname{Im} \int_{0}^{\xi} \mathrm{d} \xi_{1}=\operatorname{Im} \xi=B=\text { const }, \quad 0 \leq|B| \leq \gamma
$$

lie in the domain $D_{\xi}$ and have a positive distance from the boundary of $D_{\xi}$.

Consider a solution $I(\vartheta), \varphi(\vartheta)$ of the Hamiltonian system with real initial conditions $I(0)$, $\varphi(0)$ at $\vartheta=\vartheta_{0}=\varepsilon^{-1} \xi_{0}=0$. For

$$
I_{ \pm}=\lim _{\vartheta \rightarrow \pm \infty} I(\vartheta), \quad \Delta I=I_{+}-I_{-},
$$

referring to the paper [2], we have the estimate of $\Delta I$ that is exponentially small:

$$
\Delta I=O\left(\mathrm{e}^{-\frac{\gamma}{\varepsilon}}\right) \text {. }
$$

Actually, all variables $I, \varphi, y$ and $x$ mentioned before should contain the bar symbol "—". We obtain in fact the conclusion that

$$
\Delta \bar{I}=\bar{I}_{+}-\bar{I}_{-}=O\left(\mathrm{e}^{-\frac{\gamma}{\varepsilon}}\right), \quad \gamma=\text { const }>0 .
$$

Now return the bar. Recall that from the original Hamiltonian $E(p, q, y, x)$, we transform to two forms of Hamiltonian. With generating function $S(I, q, y, x)$ where $y, x$ are considered as parameters, we obtain $H_{0}(I, y, x)$ and with $\varepsilon^{-1} \bar{y} x+S(\bar{I}, q, \bar{y}, x)$ we obtain $H_{0}(\bar{I}, \bar{y}, \bar{x})+$ $\varepsilon H_{1}(\bar{I}, \bar{\varphi}, \bar{y}, \bar{x})$. Since the limiting values of $\bar{I}$ exist, now let us prove the lemma that the limiting value of $I$ is equal to that of $\bar{I}$ and thus exists. 


\section{Lemma 5.4.}

$$
\lim _{t \rightarrow \pm \infty} I(t)=\lim _{t \rightarrow \pm \infty} \bar{I}(t)
$$

Proof. With generating function $S(p, q, y, x)$, the Hamiltonian $E(p, q, y, x)$ is canonically transformed to $H_{0}(I, y, x)$ and $p=\frac{\partial S(I, q, y, x)}{\partial q}$.

As we know

$$
\frac{\partial S}{\partial x}=\int_{0}^{t}\left(\left\langle\frac{\partial E}{\partial x}\right\rangle-\frac{\partial E}{\partial x}\right) \mathrm{d} t_{1}
$$

So

$$
\frac{\partial S}{\partial x} \rightarrow 0, \quad \text { since } \frac{\partial E}{\partial x} \rightarrow 0, \text { as } x \rightarrow \pm \infty
$$

On the other hand, with generating function $\varepsilon^{-1} \bar{y} x+S(\bar{I}, q, \bar{y}, x)$, the Hamiltonian $E(p, q, y, x)$ is transformed to $H_{0}(\bar{I}, \bar{y}, \bar{x})+\varepsilon H_{1}(\bar{I}, \bar{\varphi}, \bar{y}, \bar{x})$ and $p=\frac{\partial S(\bar{I}, q, \bar{y}, x)}{\partial q}$. Also we know

$$
H_{1}=-\left.\frac{\partial H_{0}}{\partial \bar{x}}\right|_{\widetilde{x}} \frac{\partial S}{\partial \bar{y}}+\left.\frac{\partial E}{\partial y}\right|_{\widetilde{y}} \frac{\partial S}{\partial x},
$$

where $\widetilde{x}$ and $\widetilde{y}$ are some intermediate values. As $\frac{\partial H_{0}}{\partial \bar{x}} \rightarrow 0, \frac{\partial S}{\partial x} \rightarrow 0$, thus $H_{1} \rightarrow 0$, as $x \rightarrow \pm \infty$.

From assumption $\left(\frac{\partial H_{0}}{\partial \bar{y}}\right)^{2}+\left(\frac{\partial H_{0}}{\partial \bar{x}}\right)^{2}>$ const, and $\frac{\partial H_{0}}{\partial \bar{x}} \rightarrow 0$, as $\bar{x} \rightarrow \pm \infty$, we know that $\left|\frac{\partial H_{0}}{\partial \bar{y}}\right|>$ const for big $|\bar{x}|$. Thus, when $|\bar{x}|>c_{6},\left|\frac{\partial H_{0}}{\partial \bar{y}}\right|>c_{7}^{-1}$, where $c_{6}, c_{7}$ are positive constants. From $\dot{\bar{y}}=\frac{\mathrm{d} \bar{y}}{\mathrm{~d} t}=-\varepsilon\left(\frac{\partial H_{0}}{\partial \bar{x}}+\varepsilon \frac{\partial H_{1}}{\partial \bar{x}}\right), \dot{\bar{x}}=\frac{\mathrm{d} \bar{x}}{\mathrm{~d} t}=\varepsilon\left(\frac{\partial H_{0}}{\partial \bar{y}}+\varepsilon \frac{\partial H_{1}}{\partial \bar{y}}\right)$, the phase portrait of $H_{0}+\varepsilon H_{1}=$ const in $(\bar{x}, \bar{y})$ plane will close to that of the adiabatic approximation $H_{0}=$ const for $|\bar{x}| \leq c_{6}+1$.

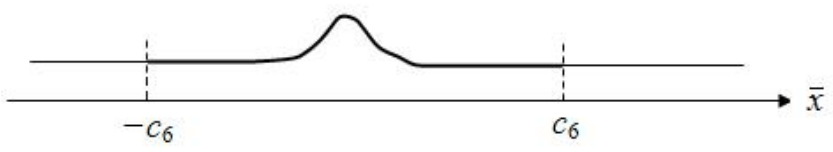

Figure 14

Thus for some time moments $t_{+}$and $t_{-}$, we have

$$
\bar{x}\left(t_{+}\right)>c_{6}, \quad \bar{x}\left(t_{-}\right)<-c_{6} .
$$


Then for $t>t_{+}$, we have

$$
\dot{\bar{x}}=\varepsilon\left(\frac{\partial H_{0}}{\partial \bar{y}}+\varepsilon \frac{\partial H_{1}}{\partial \bar{y}}\right)>\varepsilon\left(c_{7}^{-1}+O(\varepsilon)\right)>\frac{1}{2} \varepsilon c_{7}^{-1} .
$$

Therefore, $\bar{x}(t) \rightarrow+\infty$ as $t \rightarrow+\infty$. Similarly, $\bar{x}(t) \rightarrow-\infty$ as $t \rightarrow-\infty$.

From

$$
y=\bar{y}+\varepsilon \frac{\partial S(\bar{I}, q, \bar{y}, x)}{\partial x}=\bar{y}+\varepsilon V(\bar{I}, \bar{\varphi}, \bar{y}, x),
$$

taking limiting values on both sides as $t \rightarrow \pm \infty$, we obtain $y-\bar{y} \rightarrow 0$.

We also know that

$$
\begin{gathered}
p=P(I, \varphi, y, x)=P(\bar{I}, \bar{\varphi}, \bar{y}, x), \\
q=Q(I, \varphi, y, x)=Q(\bar{I}, \bar{\varphi}, \bar{y}, x) .
\end{gathered}
$$

As $y-\bar{y} \rightarrow 0$, it is evident that $I-\bar{I} \rightarrow 0, \varphi-\bar{\varphi} \rightarrow 0$ as $t \rightarrow \pm \infty$.

Therefore,

$$
\lim _{t \rightarrow \pm \infty} I(t)=\lim _{t \rightarrow \pm \infty} \bar{I}(t)
$$

Thus $I$ and $\bar{I}$ have the same limit, and $\Delta I=\Delta \bar{I}$. Therefore, we have the following corollary:

Corollary 5.2. The estimate

$$
\Delta I=O\left(\mathrm{e}^{-\frac{\gamma}{\varepsilon}}\right)
$$

is valid.

\section{Acknowledgements}

I am glad to express my gratitude to Professor Anatoly Neishtadt for providing detailed comments and frequent discussion during the research and completion of this article. 


\section{References}

[1] Arnold, V.I., Kozlov, V.V. and Neishtadt, A.I.: Mathematical Aspects of Classical and Celestial Mechanics. Second augmented and revised edition. Springer-Verlag, 2006.

[2] Neishtadt, A.I.: On the Accuracy of Persistence of Adiabatic Invariant in Single-Frequency Systems. Regular and Chaotic Dynamics, Vol. 5, No 2, 2000, pp. 213-218.

[3] Arnold, V.I.: Mathematical Methods of Classical Mechanics. Second Edition. Springer, 2000.

[4] Neishtadt, A.I.: On the Change in the Adiabatic Invariant on Crossing a Separatrix in System with Two Degrees of Freedom. PMM U.S.S.R, Vol. 51, No. 5, 1987, pp. 586-592.

[5] Brown, J.W. and Churchill, R.V.: Complex Variables and Applications. Sixth Edition. McGraw-Hill, 1996.

[6] Arnold, V.I.: Ordinary Differential Equations. The MIT Press, Cambridge, Massachusetts and London, 1973.

[7] Benettin, G., Carati, A. and Gallavotti, G.: A Rigorous Implementation of the Jeans-Landau-Teller Approximation for adiabatic invariants. Nonlinearity 10, 1997, pp. 479-505.

[8] Treschev, D.V.: The Method of Continuous Averaging in the Problem of Fast and Slow Motions. Regular and Chaotic Dynamics, Vol. 12, No. 3/4, 1997, pp. 9-20 